\title{
Criticality of Rupture Dynamics in 3-D
}

\author{
Raul Madariaga ${ }^{1}$ and Kim B. Olsen ${ }^{2}$
}

\begin{abstract}
We study the propagation of seismic ruptures along a fault surface using a fourth-order finite difference program. When prestress is uniform, rupture propagation is simple but presents essential differences with the circular self-similar shear crack models of Kostrov. The best known is that rupture can only start from a finite initial patch (or asperity). The other is that the rupture front becomes elongated in the in-plane direction. Finally, if the initial stress is sufficiently high, the rupture front in the in-plane direction becomes super-shear and the rupture front develops a couple of "ears" in the in-plane direction. We show that we can understand these features in terms of single nondimensional parameter $\kappa$ that is roughly the ratio of available strain energy to energy release rate. For low values of $\kappa$ rupture does not occur because Griffith's criterion is not satisfied. A bifurcation occurs when $\kappa$ is larger than a certain critical value, $\kappa_{c}$. For even larger values of $\kappa$ rupture jumps to super-shear speeds. We then carefully study spontaneous rupture propagation along a long strike-slip fault and along a rectangular asperity. As for the simple uniform fault, we observe three regimes: no rupture for subcritical values of $\kappa$, sub-shear speeds for a narrow range of supercritical values of $\kappa$, and super-shear speeds for $\kappa>1.3 \kappa_{c}$. Thus, there seems to be a certain universality in the behavior of seismic ruptures.
\end{abstract}

Key words: Rupture dynamics, finite-difference modeling, spontaneous rupture propagation.

\section{Introduction}

Earthquakes are complex at all scales. Recent studies of a number of earthquakes that were well-recorded in the near field reveal that both slip and stress drop in these earthquakes are very complex. The first evidences of these complexities were discussed by DAS and AKI (1977a), AKI (1979), DAY (1982) and many others. Beroza and Mikumo (1996), Bouchon (1997), Ide and TAKeo (1997), among others, showed that stress drop for all well studied earthquakes is highly variable in space and can be both positive (where fault slips) or negative (stress increases) where there is no slip. Some of these properties were indeed introduced in the original "asperity" model of KANAMORI and STEWART (1976) and in the "barrier" model of DAS and AKI (1977b) who realized that the simple uniform stress drop

\footnotetext{
${ }^{1}$ Laboratoire de Géologie, Ecole Normale Supérieure, 24 rue Lhomond, 75231 Paris Cedex 05, France. E-mail: madariag@geologie.ens.fr

${ }^{2}$ Institute for Crustal Studies, University of California, Santa Barbara, CA 93106, U.S.A. E-mail: kbolsen@crustal.ucsb.edu
} 
models were exceedingly simple representations of the earthquake source. MADARIAGA (1979) showed that seismic radiation from these models would be very complex and that high frequency radiation would be quite different from that predicted by the simple circular crack models of Kostrov (1964) and MADARIAGA (1976).

The study of complex fault models has gained new attention in recent years in the wake of the suggestion by CARLSON and LANGER (1989) that earthquakes may become spontaneously complex due to nonlinear effects in friction. Other alternatives to the origin of complexity were discussed by RICE (1993) and RICE and BEN-ZION (1996), who suggested that at least some of the complexity found by Carlson and Langer was due to the lack of a continuum limit in the velocity weakening friction law they used. Applying a simple regularized friction law with velocity weakening, CochARD and MADARIAGA (1996) found that heterogeneity appears spontaneously only in a limited parameter range of rate-weakening. More recently, SHAW and RICE (2000) have studied the conditions for the development of complexity in well-posed numerical simulations.

In order to study complexity we must model earthquakes accurately, taking into account all the relevant length scales in the problem, both (intrinsic) length scales associated with friction as well the (extrinsic) scale associated with fault size and asperity distribution. This is not a simple task because accurate numerical modeling of ruptures requires vast amounts of computer resources. Although DAS and Kostrov (1983) and DAY (1982) made calculations of complex faults with heterogeneous distributions of stress and rupture resistance, these models contained insufficient resolution to study the more difficult problem of the interaction between intrinsic and extrinsic length scales. Recent development of finite difference methods in 3-D (Harris and Day, 1993; Mikumo and Miyatake, 1995; Olsen et al., 1997) or Boundary Integral Equation methods (FUKUYAMA and MADARIAGA, 1998), together with the availability of fast parallel computers, has opened the way to the study of accurate rupture propagation models.

In this paper we will report on our study of the nature of three-dimensional rupture propagation for a couple of very simple classical models of rupture: the rectangular fault and rectangular asperity. With our new computational capabilities we study the propagation of rupture in these models for a broad parameter range and demonstrate that rupture propagation is controlled by a simple nondimensional number. The nondimensional number has a critical value below which ruptures die very quickly. For nondimensional values slightly above critical, ruptures grow at speeds close to the shear wave speed as most earthquakes do. For larger values, ruptures grow faster than the speed of shear waves, which is rarely observed unambiguously in earthquakes. By examining the stress field of recent events we suggest that earthquake ruptures occur at nondimensional numbers that are most often critical. Although we cannot prove it for the moment, we believe that this may be the actual self-organized criticality of earthquake ruptures. 
An essential requirement to study dynamic faulting is an accurate and robust method for the numerical modeling of seismic sources. In our recent work we have used a fourth-order formulation of the velocity-stress method (MADARIAGA, 1976; Olsen et al., 1997; MADARIAGA et al., 1998) in order to study dynamic rupture propagation on a planar shear fault embedded in a heterogeneous elastic half-space.

Rupture propagation on a major earthquake fault is controlled by the properties of the friction law on the fault. Friction controls the initiation, development of rupture and the healing of faults. Laboratory experiments at low slip rates were analyzed by DIETERICH (1978), who proposed models of rate- and state-dependent friction, and by OHNAKA and SHEN (1999) who concluded that their experiments could be explained with a simpler slip-weakening friction law. Actually, from the point of view of earthquake observations, these two models of friction are essentially indistinguishable as shown by ОкUво (1989). Basically, both slip-weakening and rate- and state-dependent friction contain finite length scales that control the behavior of the rupture front. Although, these intrinsic length scales are of very different origin, we can hypothesize that their effects on rupture is similar. The length scale, that we will generically refer to as $D_{c}$, is the most important ingredient in our study. Without this length scale earthquake faulting makes little sense due to lack of energy conservation in the fault system.

Because of the equivalence of friction laws at high slip rates, we used a simple slip-weakening friction law in which slip is zero until the total stress reaches a peak value (yield stress) that we denote with $T_{u}$. Once this stress has been reached, slip $D$ increases and $T(D)$ decreases:

$$
\begin{array}{lll}
T(D)=T_{u}\left(1-\frac{D}{D_{c}}\right) & \text { for } & D<D_{c} \\
T(D)=0 & \text { for } & D>D_{c}
\end{array}
$$

where $D_{c}$ is a characteristic slip distance. This friction law has been applied in numerical simulations of rupture by ANDREWs (1976), DAY (1982) and many others. We note that in (1) and in the following we refer all our stresses to a reference value equal to the residual friction at high slip. This is why $T(D)=0$ in the second equation of (1).

Figure 1 presents the geometry of the fault model we study. The most important feature of the friction law is slip-weakening that occurs near the rupture zone on a so-called breakdown or slip-weakening zone just behind the rupture front. The propagation of the rupture front is completely controlled by the friction law and the distribution of initial stress on the fault. 


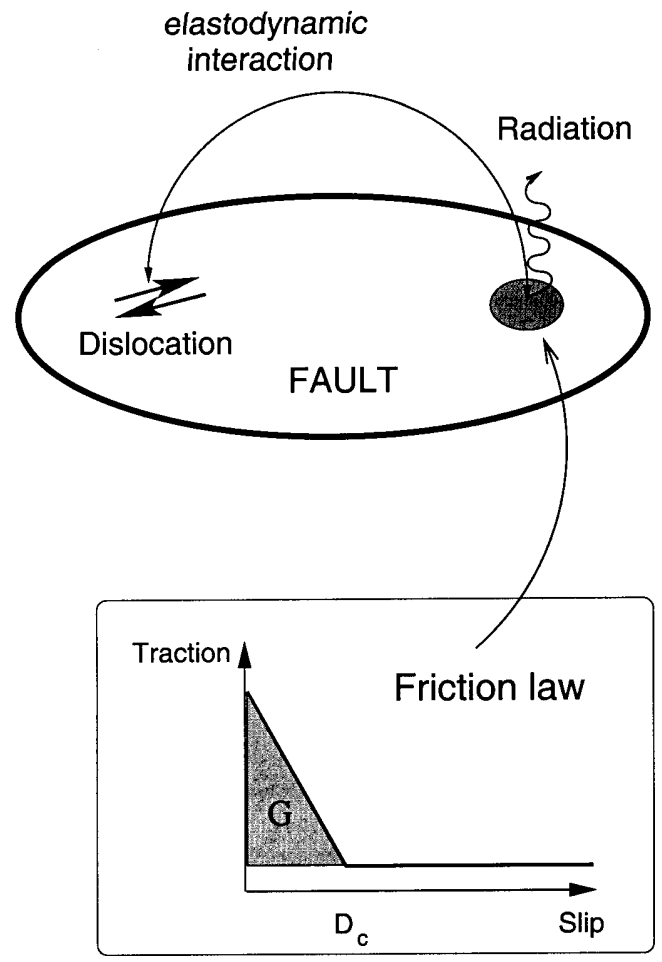

Figure 1

Geometry of a simple rupture propagating along a flat fault embedded in a 3-D elastic medium. Rupture propagation is controlled by elastodynamic interactions and is damped by seismic radiation. The boundary conditions on the fault are dominated by friction. We use a simple slip-weakening friction of peak stress $T_{u}$, slip-weakening $D_{c}$ and energy release rate $G$.

\section{Spontaneous Rupture of a Uniform Fault}

MADARIAGA et al. (1998) studied spontaneous rupture, starting from a circular asperity of radius $R$ that is ready to break (with stress $T_{u}$ ), and is surrounded by a fault surface at a constant effective stress level $\left(T_{e}<T_{u}\right)$. Rupture is initiated at time $t=0$ by instantaneously triggering stress relaxation inside the asperity of radius $R$ according to the friction law of equation (1). This instantaneous rupture of the circular patch is less physical than starting from a critical fault and letting the rupture grow spontaneously. Unfortunately, rupture of a critical fault is an expensive numerical problem because rupture must start from one point on the edge, encircle the asperity and then grow outside the initial patch. Such a problem would take excessive time to solve in our currently available computer.

As ANDREws (1976) and many other authors have noted, in order for rupture to expand stress must be high over a finite zone, sometimes referred to as the minimum rupture patch. Once rupture has broken the asperity, it will grow or stop 
depending on the values of the stress field inside $\left(T_{u}\right)$ and outside the asperity $\left(T_{e}\right)$, the shear modulus $\mu$, the half-width of the asperity $L=R$ and the slip-weakening constant $D_{c}$ of the friction law in (1).

ANDREWs (1976) computed the minimum patch for a 2-D in-plane fault from energy considerations and found that the critical half-width of the fault was:

$$
L_{c}=\frac{1}{\pi(1-v)} \frac{T_{u} \mu}{T_{e}^{2}} D_{c},
$$

where $v$ is Poisson's modulus. He derived this relationship by equating the available strain energy in the vicinity of the fault to the energy needed to propagate rupture (i.e., $G=1 / 2 T_{u} D_{c}$ ) at one edge of the fault. A similar expression for a circular fault was computed by DAY (1982) who found that the minimum radius of the initial patch was:

$$
R_{c}=\frac{7 \pi}{24} \frac{T_{u} \mu}{T_{e}^{2}} D_{c} .
$$

It is well-known in fluid mechanics (see, e.g., LANDAU and LIFSHITZ, 1959) that expressions like (2) and (3) can also be derived from dimensional analysis. In doing that we introduce a nondimensional number $\kappa$ defined as follows:

$$
\kappa=\frac{T_{e}^{2}}{\mu T_{u}} \frac{L}{D_{c}},
$$

where $L$ is a characteristic length scale, for instance the half-width of the fault, and $T_{e}, T_{u}, \mu$ and $D_{c}$ were defined earlier. These are the only parameters in this problem, thus there is no need to introduce additional nondimensional numbers to study the bifurcation.

When considered as a function of $L$, expression (4) together with (2) or (3) defines a bifurcation at a certain critical value $K_{c}$, so that ruptures grow only for values of $L \geq L_{c}$. For in-plane faults $\kappa_{c}$ can be derived from ANDREWs (1976) expression reproduced in equation (2). We obtain $\kappa_{c}=1 /[\pi(1-v)] \simeq 0.424$ for $v=1 / 4$, the usual value of Poisson's ratio for crustal materials. For anti-plane rupture the minimum patch can be computed from IDA's (1972) expressions, we find $\kappa_{c}=1 / \pi=0.318$. Thus, as expected, it is easier to initiate shear fracture in anti-plane mode than in in-plane mode. For a circular fault the critical number can be estimated from (3) as $\kappa_{c}=7 \pi / 24=0.916$. From numerical simulations MADARIAGA et al. (1998) found a $\kappa_{c} \simeq 0.6$. The difference probably comes from the loading conditions assumed by DAY (1982) who studied the rupture of a quasi-statically loaded crack, while MADARIAGA et al. (1998) triggered rupture by suddenly loading a circular asperity.

We realize that $\kappa_{c}$ defines a typical Hopf bifurcation. For $\kappa<\kappa_{c}$ rupture does not grow beyond the initial asperity; while for $\kappa>\kappa_{c}$ it grows indefinitely at increasing speed without ever stopping. This is a typical bifurcation first noted by 
GRIFFITH (1929). However there is a further complication: if the parameter $\kappa$ is considerably larger than $\kappa_{c}$ the rupture front in the in-plane direction jumps to speeds higher than the shear-wave speed. The jump to super-shear speeds was first reported for plane faults by ANDREws (1976), who attributed it to the interaction between an $S$ wave running ahead of the crack and the rupture front itself. In MADARIAGA et al. (2000) we show that in 3-D the jump from sub-Rayleigh to super-shear speeds is due to an instability of the rupture front that spreads along the rupture front like a wave. For the initially circular shear fault, the rupture front acquires a very nice elliptical shape with two "ears" elongated along the in-plane direction (see the bottom snapshots in Figure 2).

Rupture in the simple uniform model studied here will never stop once it starts. Stopping ruptures in 2-D was discussed by Husseini et al. (1975) who showed that ruptures would stop either because they met unbreakable barriers, or because they ran out of steam when they entered regions with low stress (a sort of anti-asperity). In 3-D there are many other possibilities for rupture to stop because stress distributions can be very complex as mentioned above.

\section{Very Long Rectangular Barrier Model on a Flat Fault}

We study two simple models that will put in evidence a simple nondimensional number that controls rupture growth in 3-D. In order to understand rupture we must introduce external length scales. These length scales are naturally associated either with the distribution of rupture resistance in the "barrier" model or with the distribution of initial stress in the "asperity" model. We will demonstrate that although the same nondimensional number controls rupture processes in both models, the critical value of the bifurcation differs.

We study first a model of faulting with only one external length scale. We assume that rupture is guided by the presence of two strong barriers defining a long rectangular fault zone (see the upper part of Fig. 3). Rupture resistance is constant $\left(T_{u}=1\right)$ inside a zone of width $W$. Outside this zone rupture resistance is sufficiently high (effectively $\infty$ ), so that ruptures never break outside the fault zone. For numerical reasons and to avoid end effects we also put barriers along the direction of faulting. We assume that the entire fault plane is subject to constant "effective" shear stress $T_{e}$. The only additional ingredient in our model is the friction law defined above (1). This problem has two stress parameters $T_{u}$ and $T_{e}$ although only their ratio is relevant to rupture. Two length scales are involved: $W$, the half-width of the fault and $D_{c}$, the slip-weakening distance of the friction law. Using the same dimensional arguments that led to (4), we define the nondimensional number

$$
\kappa=\frac{T_{e}^{2}}{\mu T_{u}} \frac{W}{D_{c}},
$$


Slip Rate

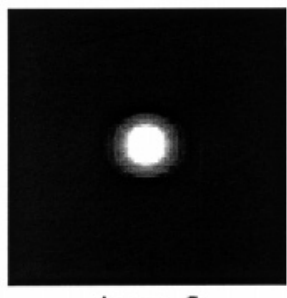

$\mathrm{t}=6$

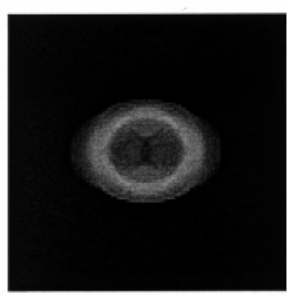

$t=12$

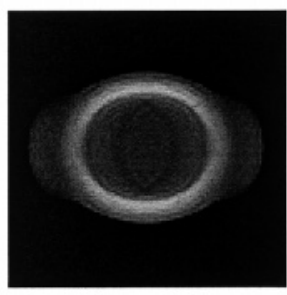

$t=18$

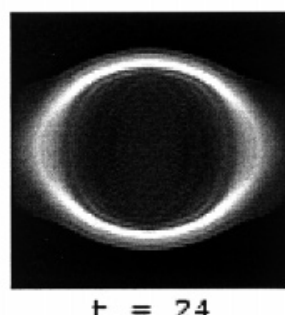

$t=24$

\section{Shear Stress}

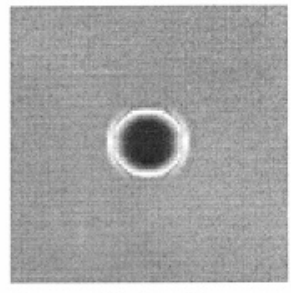

$t=6$

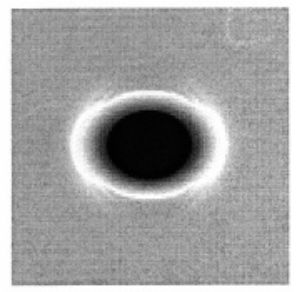

$t=12$

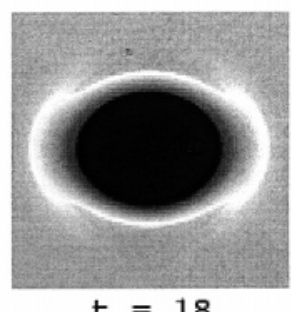

$\mathrm{t}=18$

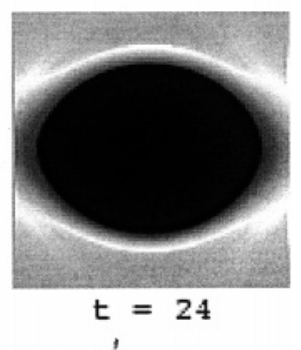

\section{$0 \quad$ Non-dimensional units 1}

Figure 2

Rupture growth on a flat perfectly uniform fault embedded in a homogeneous elastic medium. Rupture starts from a finite initial asperity and then grows at subsonic speed in all spatial directions. After an interim rupture along the in-plane direction jumps at a speed that is higher than that of shear waves. The snapshots on the left show slip rate on the fault; and those on the right show the corresponding shear stress field. Snapshots are shown at four successive instants of time. Time is measured in units of time for a shear wave to propagate across the asperity. Around time 18, rupture jumps to super-shear speed along the in-plane direction (horizontal axis). 


\section{Rectangular Barrier Model}

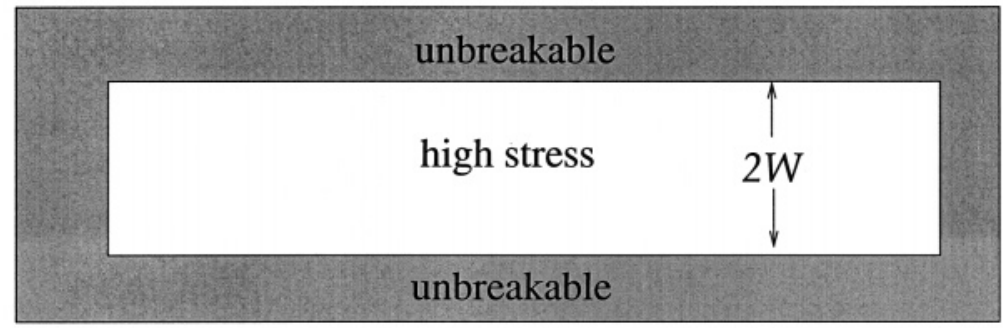

\section{Rectangular Asperity Model}

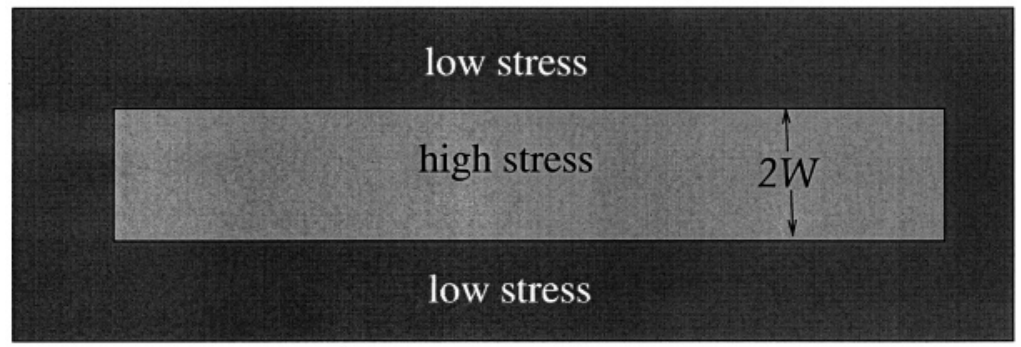

Figure 3

Two models of simple dynamic shear faults studied in this paper. (top) A rectangular fault loaded by uniform stress and bounded by unbreakable barriers. (bottom) A rectangular asperity where rupture resistance is uniform but initial stress is concentrated in a band or asperity.

in which we have replaced $L$ by the half-width $W$ of the fault. We will now show that this nondimensional number completely controls rupture propagation.

In the numerical simulations we surrounded the fault by a finite homogeneous elastic medium with absorbing boundary conditions. As in the unbounded fault plane, we triggered rupture by instantaneously starting slip on a circular patch of radius $R$. This radius was fixed in our computations $(R=W / 2)$. It is smaller than $W$ because as will be shown below the critical number for the initiation of rupture from a circular patch is always less than the critical number for propagation along a rectangular fault. All computations were done with $W=48$ grid points.

In Figure 4 we present snapshots of the slip rate and shear stress fields on the fault at the same instant of time $t=5 \mathrm{~W} / \beta$. All these snapshots were calculated with a value of $D_{c}=0.416 W T_{u} / \mu$. The control parameter for these calculations was the ratio $T_{e} / T_{u}$ that varied from 0.55 on the top panel of Figure 4 up to 0.7 at the bottom. In the top panel $(\kappa=0.726)$ rupture has already stopped at time $t=5 \mathrm{~W} / \beta$. This shows clearly that seismic ruptures can stop spontaneously. The stress field 
shown on the right hand of the top panel is very close to the final static stress change around the ruptured part of the fault. In the middle panels $(\kappa=0.864)$ we show the results for a fault that is very close to critical. Here, rupture advances at subsonic speed along the longitudinal direction of the fault. The rupture front in this model is almost steady state and will simply continue to propagate forever at this speed unless it encounters stress or frictional heterogeneity. The slip rate indicates that the width of the rupture front is probably controlled by the fault width, as suggested by DAY (1982). The stress field on the right-hand side manifests a peak in stress that propagates slightly ahead of the rupture front. This is the shear-wave front that establishes that rupture is sub-shear in this case. Finally in the bottom two panels $(\kappa=1.176)$ we show slip rate and stress change for a super-critical rupture. Here the rupture front has jumped ahead of the shear-wave front and is close to a steady state. Rupture will continue forever at super-shear speeds unless it encounters stress or strength heterogeneities.

The results shown in Figure 4 are clearly reminiscent of a bifurcation in the behavior of the system for a particular value of the effective stress $T_{e} / T_{u}$. These results were found for a particular value of the slip-weakening distance $D_{c} / W=$

\section{Rectangular Fault $\quad$ W/Dc $=2.4$}

Slip Rate

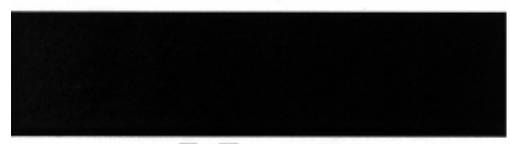

$\mathrm{Te} / \mathrm{Tu}=0.55$

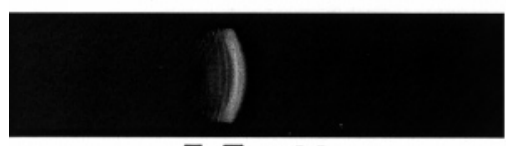

$\mathrm{Te} / \mathrm{Tu}=0.6$

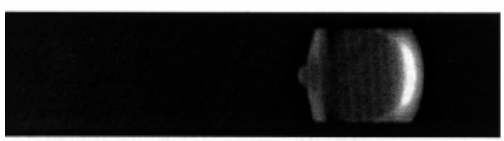

$\mathrm{Te} / \mathrm{Tu}=0.7$
Shear Stress

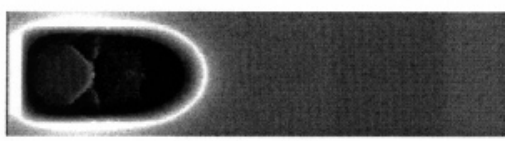

$\mathrm{Te} / \mathrm{Tu}=0.55$
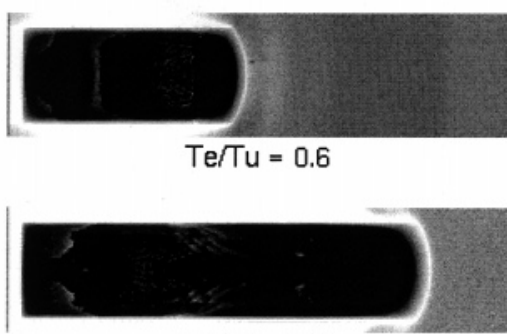

$\mathrm{Te} / \mathrm{Tu}=0.7$

$0 \quad$ Non-dimensional units 1

Figure 4

Snapshots of the shear stress (right) and slip rate (left) fields on a fault bounded by two unbreakable barriers as shown in Figure 3. The critical value for this configuration is $\kappa_{c}=0.8$ as shown in Figure 5. The top panels show ruptures at subcritical $(\kappa=0.726)$, slightly super critical $(\kappa=0.864)$ and very super critical $(\kappa=1.176)$ values of the nondimensional parameter $\kappa$. 


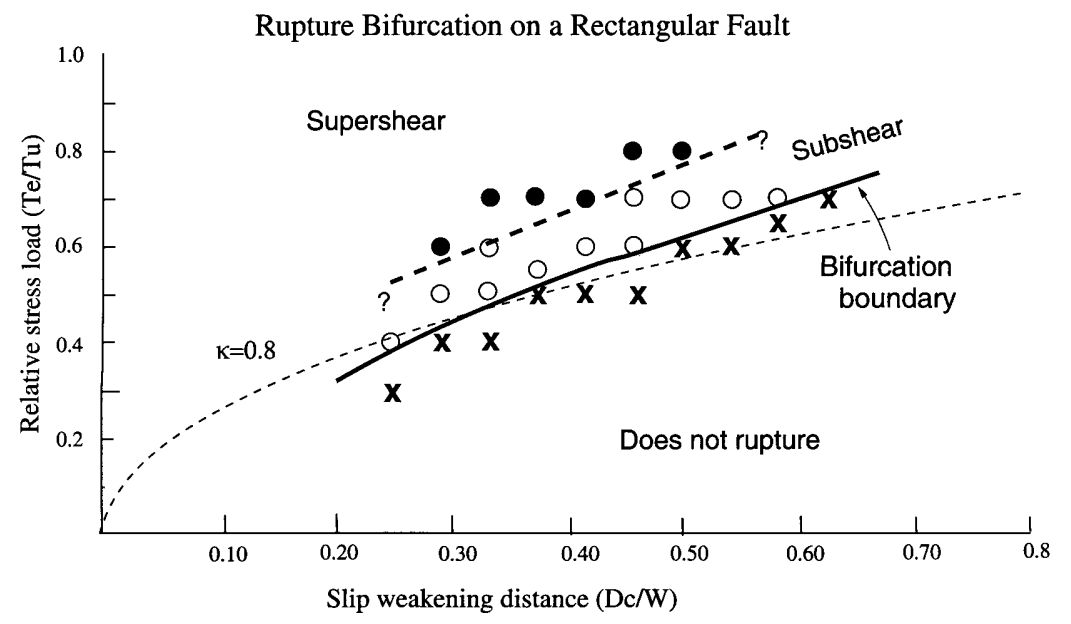

Figure 5

Bifurcation conditions for the growth of a rectangular fault. The crosses represent parameter values for which rupture stops spontaneously. The empty circles are parameter values for which rupture propagates along the fault with a rupture velocity below the shear-wave speed. Filled circles are parameter combinations that produce super-shear fracture. The thick continuous and discontinuous lines separate these parameter fields. The thin dashed line is the approximate critical value $\kappa_{c}=0.8$ and corresponds very well with the thick line, the critical boundary found experimentally.

$0.416 T_{u} / \mu$; changing this ratio affects the value of $T_{e} / T_{u}$ at which the bifurcation between non-growth and growth occurs.

In order to explore the conditions of criticality in this model, we carried out numerous simulations varying the ratios $D_{c} / W$ and $T_{e} / T_{u}$. In Figure 5 we present the results of our study. Numerical computations are reproducible and feasible only in the range shown. For $D_{c} / W<0.30$ the accuracy of the rupture front resolution is inadequate (see MADARIAGA et al., 1998) and for $D_{c}>0.7 \mathrm{~W}$, faulting is not well resolved across the fault width. Thus we expect strong end effects at low and large values of $D_{c} / W$. These limitations could be reduced by using a denser numerical grid. However, there is a clear systematic separation between the region of growth and non-growth indicated by the thick line in Figure 5, and a similar boundary between sub-shear and super-shear fracture further into the zone of rupture, as indicated by the thick, dashed line. We can approximate the critical boundary using the expression (5) with a value of $\kappa=0.8$. This is indicated by the thin dashed lined marked with the value $\kappa=0.8$. This is the critical value for this particular model of rupture, although as remarked earlier its exact value can only be approximately determined because of end effects. We note that it is larger than the critical value we inferred for the circular fault $\left(\kappa_{c}=0.6\right)$. This explains why it is sufficient to start the rupture from a circular patch of radius $R=W / 2$. 


\section{Very Long Rectangular Asperity on a Flat Fault}

In order to study the condition for rupture propagation in a heterogeneous initial stress field, we turn to a very simple rectangular asperity model described in the bottom panel of Figure 3. In this model the initial stress field contains a long asperity of width $W$ loaded with a longitudinal "effective" shear stress $T_{e}$. The asperity is surrounded by a fault plane in which stress is very low (only $0.1 * T_{u}$ ). At time $t=0$ rupture is initiated by forcing rupture over a circular patch of radius $R>R_{c}$ where $R_{c}$ is the critical size for the circular asperity discussed earlier in the paper. Depending on the value of $T_{e}$, and the slip-weakening distance $D_{c}$, rupture either grows along the asperity or stops very rapidly. As for the rectangular fault we again have a bifurcation in rupture behavior, controlled by a critical value.

In Figure 6 we show the slip rate and stress fields on the fault at the same instant of time $t=5 \mathrm{~W} / \beta$, for three values of the load $T_{e} / T_{u}$. In all these simulations the slip-weakening distance was kept fixed at $D_{c} / W=0.833 T_{u} / \mu$. On the top right we show the results for $T_{e} / T_{u}=0.7$. Rupture started in this case near the left end of the asperity and stopped growing very rapidly. At time $t=5 \mathrm{~W} / \beta$ rupture has already stopped and what we see on the right-hand panel is the static shear stress field left over by the rupture. The second row shows the results obtained for

\section{Rectangular Asperity $\quad \mathrm{W} / \mathrm{Dc}=1.2$}

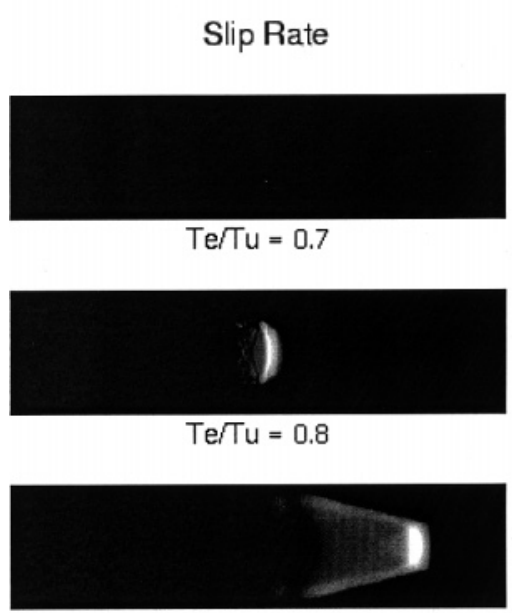

$\mathrm{Te} / \mathrm{Tu}=0.9$
Shear Stress

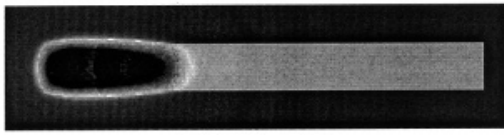

$\mathrm{Te} / \mathrm{Tu}=0.7$

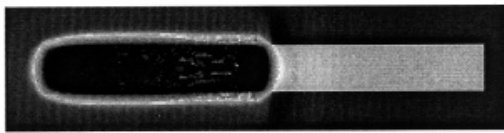

$\mathrm{Te} / \mathrm{Tu}=0.8$

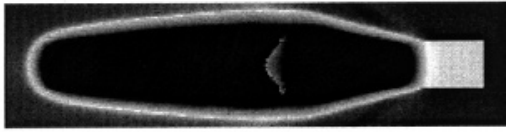

$\mathrm{Te} / \mathrm{Tu}=0.9$

$0 \quad$ Non-dimensional units 1

Figure 6

Snapshots of the shear stress and slip rate fields on a fault containing a very long and narrow asperity (clear in the stress snapshots). The top panels show ruptures at subcritical $(\kappa=0.588)$, slightly super critical $(\kappa=0.768)$ and very super critical $(\kappa=0.972)$ values of the parameter $\kappa$. 
$T_{e} / T_{u}=0.8$, where rupture propagates along the asperity at sub-shear speeds. We see that the rupture zone extends beyond the asperity, leaving an elongated final fault shape. Finally, at the bottom of Figure 6 we show snapshots of slip rate and stress for a super-critically loaded asperity with $T_{e} / T_{u}=0.9$. In this simulation the rupture front is running faster than the shear wave, producing a wake that spreads deeply into the lower prestress zone. Thus, as for the rectangular fault model, we are again in the presence of a bifurcation: when $\kappa$ is smaller than critical as in the case in the top panels of Figure 6, rupture stops. When rupture is lightly super-critical as in the central panel, rupture grows slowly at speeds close to the shear-wave velocity. Finally, for very super-critical conditions, as in the bottom panels, rupture becomes faster than the shear wave speed, producing strong super-shear head waves in the low stress areas that surround the asperity.

For the rectangular asperity model we omitted the systematic exploration of the parameter space that we carried out for the rectangular fault (Fig. 5). Such parameter study is computationally expensive and we expect the effects to be similar. The sole difference between the asperity is lower for the former than for the latter. We find $\kappa_{c}=0.7$ for the asperity model which may be compared to 0.8 for the barrier model. This is expected since an asperity compared to a barrier of the same dimensions is easier to break because rupture will extend into the low stress areas surrounding the asperity. This is of course impossible in the barrier model. Finally, as shown in Figure 6, we find again that for large enough $\kappa$ ruptures grow initially at very high speeds and then jump to a speed higher than the shear-wave velocity.

Although many authors have studied rupture conditions in the presence of asperities and barriers, most notably DAS (1981), DAS and Kostrov (1983), Kostrov and DAS (1989) and DAY (1982), we believe that this is the first time that conditions for rupture growth and arrest are systematically studied in a simple 3-D rupture model. Our results are entirely compatible with and extend those of HUSSEINI et al. (1975) who studied conditions for rupture of 2-D faults. As we will show the nondimensional number $\kappa$ is closely related to the minimum patch that appears for all friction laws containing a length scale (see the pioneering work by IDA, 1972; ANDREWS, 1976; DAY, 1982).

\section{Spontaneous Rupture on a Realistic Fault: The Landers 1992 Earthquake}

OLSEN et al. (1997) generated a fully dynamic rupture model of the Landers earthquake of July 1992 that was based on the kinematic model inverted by WALD and Heaton (1994) using a combination of seismic and geodetic data with minimum wavelengths of 3-4 km. From the kinematic slip model they computed the stress drop on the fault and found that it was very heterogeneous, containing both large positive stress drops where the fault slipped and very negative values 
where it did not slip. They generated from this stress drop field an initial stress field that is shown in the top panel of Figure 7, superimposed with the patch in the upper right part of the fault where rupture was initiated in the simulation. The range of variation of initial stress in this and the following panels of Figure 7 is roughly from $-12 \mathrm{MPa}$ to $12 \mathrm{MPa}$. In addition to the initial stress field, the only other assumption in the dynamic simulation is that the friction law (1) was the same everywhere on the fault plane. After trial and error modeling, OLSEN et al. (1997) fixed $T_{u}$ at $12 \mathrm{MPa}$, which approaches the maximum value of the initial stress field shown in the first panel of Figure 7. The only free parameter that was left to be determined was the slip-weakening distance $D_{c}$. OLSEN et al. found that rupture would be similar to the kinematic one only if $D_{c}$ was close to $0.8 \mathrm{~m}$. For smaller values the rupture would break the fault at super-shear speed, leaving a uniform final stress field, and a slip distribution that was completely different from that of WALD and HEATON (1994). For larger $D_{c}$, on the other hand, rupture would simply not propagate along the fault. Thus again, we are dealing with a critical phenomenon, as in the two previous examples discussed above.

We can make a quick calculation of the value of $\kappa$ for the OLSEN et al. model. As we mentioned above, $T_{u}=12 \mathrm{MPa}$ and $D_{c}=0.8 \mathrm{~m}$ so that the energy release rate $G=4.8 \mathrm{MJm}^{-2}$. This is a large value but on the order of magnitude of those proposed by AKI (1979) and OHNAKA and SHEN (1999). In order to compute $\kappa$ we need an average value for $T_{e}$ which is estimated from the initial stress field to be 4 MPa (see MAdARIAGA, 1979 for a discussion of different ways to compute the average stress drop). Then, using the average value $\mu=3.45 \times 10^{10} \mathrm{~Pa}$ and a fault half width of $15 \mathrm{~km}$, we find $\kappa=0.72$ for the OLSEN et al. (1997) Landers earthquake model. We note that in order to estimate $\kappa$ for Landers we used a fault half-width of $15 \mathrm{~km}$ instead of $7.5 \mathrm{~km}$ in order to take into account approximately the effect of the free surface. $\kappa$ for the Landers earthquake is well below the critical values we obtained above for the rectangular fault models $(\kappa=0.8)$. This is not surprising since the rupture in Landers propagated mainly on those parts of the fault where stresses were close to the maximum of $T_{e}=10 \mathrm{MPa}$. All this seems to indicate that rupture in the Landers earthquake reproduces the general behavior of the kinematic model of WALD and HEATON only if $\kappa$ is very close to critical. There are many corrections applicable to this calculation, however we believe that the main result will stand further scrutiny: rupture in the Landers earthquake was very close to critical, enough to make the rupture grow but not enough for the rupture to become super-shear.

The result that the Landers earthquake rupture was close to critical may be obtained in another way. Careful study of rupture propagation in the initial stress field shown at the top of Figure 7 reveals that rupture only propagates in regions of high stress and sufficient width. It takes a very minor modification of the initial stress field to either stop it or guide it into a different area of the fault. 


\section{Stress change}
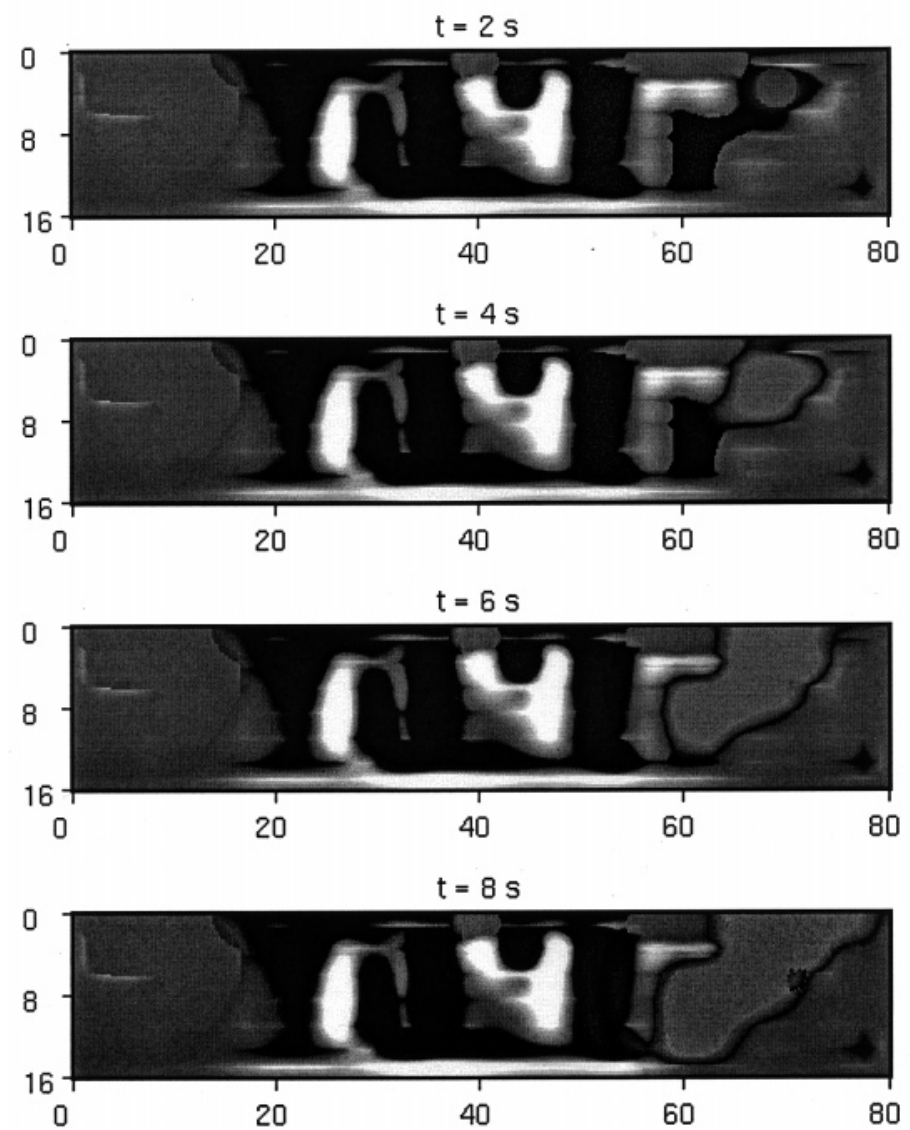

$t=10 s$

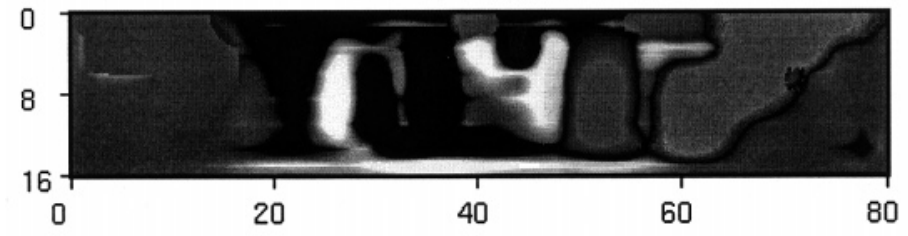

$\mathrm{t}=12 \mathrm{~s}$

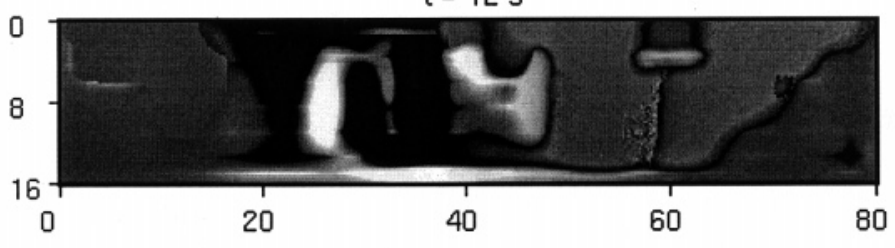

$-12 \quad$ stress (Mpa) 
In Figures 7 and 8 we show the longitudinal shear stress and slip rate fields at several successive instants of time at $2 \mathrm{~s}$ intervals. Only the initial instants of rupture simulation are shown here. Note that slip rate is faint and weak. As proposed by HeAton (1990) the instantaneous rupture width seems not to be controlled by the overall width of the fault but by the size of the local asperities or concentrations of the initial stress distribution on the fault. This is in agreement with the observations of BEROZA and Mikumo (1996) and with theoretical results of Cochard and MADARIAGa (1994). Rupture in Figure 8 is never super-shear, it barely makes it through the fault just as one would expect from a critical phenomenon.

Next we analyzed the details of rupture. The stress field is not very favorable to rupture initiation because the stresses in the vicinity of the initial patch are relatively weak. Therefore as shown in Figures 7 and 8, the initial rupture takes a long time before reaching the vertical high stress zone to the northwest (left) of the initial patch. Upon entering this zone, rupture propagates downwards at high speeds because the loaded zone is relatively wide. Upon hitting the bottom of the fault it stops because there is no lateral communication in this area.

As a final remark concerning criticality, we observe that rupture can initiate from many points on the fault for the initial stress field shown in Figure 7. We have generated models of rupture that start from any high stress spot on the fault, all indicating that the final stress and slip distributions were almost insensitive to the actual kinematics of rupture. As long as we stay close to critical conditions rupture seems to be controlled mainly by the initial stress field. We have yet to complete the study of rupture in Landers although we expect that we can control the way rupture grows by simple manipulation of the initial stress field (the kinematics).

In conclusion, the initial stress field controls rupture propagation very closely. As long as rupture conditions are very close to critical, rupture extends following a relatively clear pattern of infiltration. It penetrates places where stress is high over large patches and completely avoids the zones where stresses are low. The overall kinematics are then similar to those determined by WALD and HeAton (1994), CoHeE and Beroza (1994) or Cotton and CAMPillo (1995).

\section{Discussion: Where Does the Nondimensional Number Come from?}

Determining the origin of the nondimensional number that controls the bifurcation is a complex problem that will require the study of many models with other

Snapshots of the shear stress field on the fault of the Landers 1992 earthquake. Snapshots were calculated at regular intervals of $2 \mathrm{~s}$. The initial shear stress is shown in the upper snapshot, modified by the stress drop shortly after rupture initiation. 
Slip rate
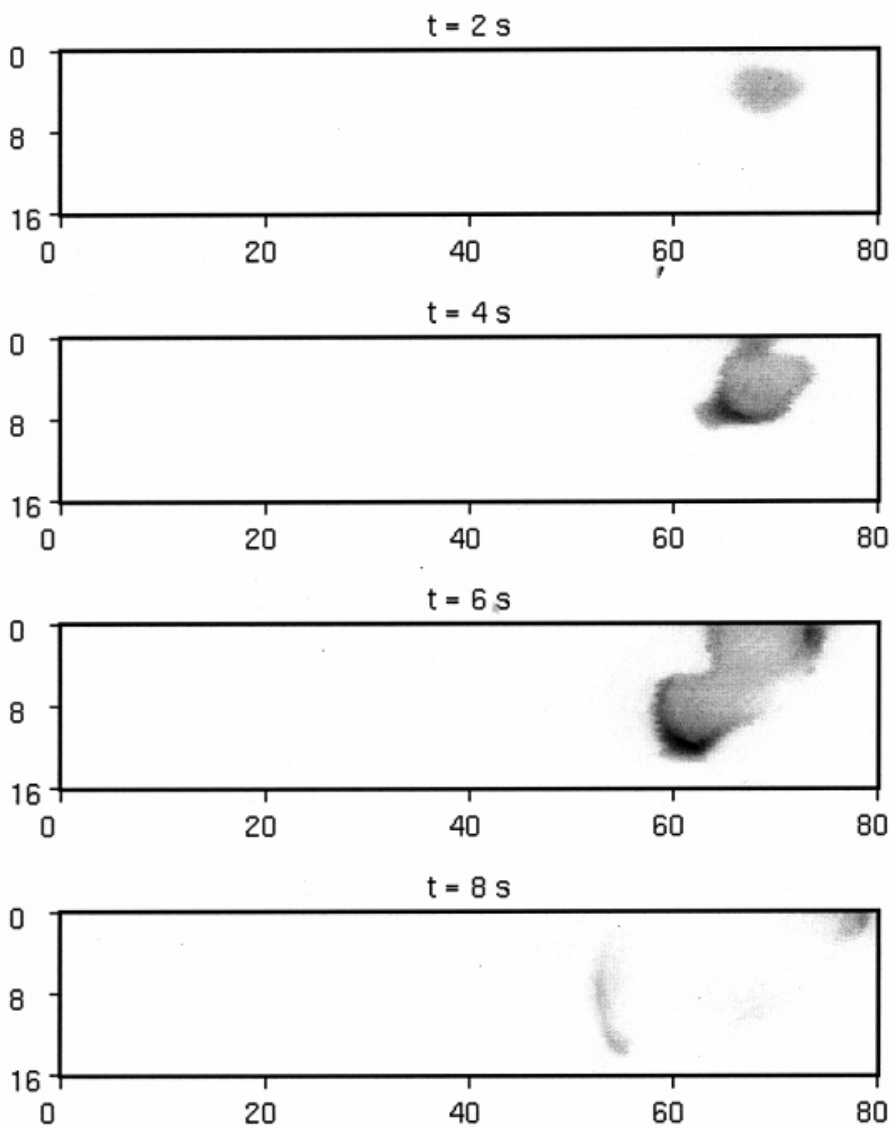

$t=10 s$

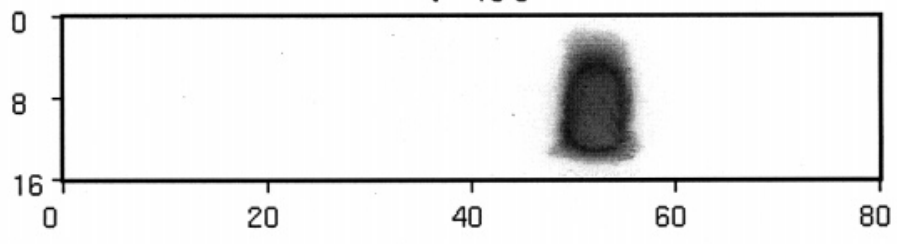

$t=12 s$

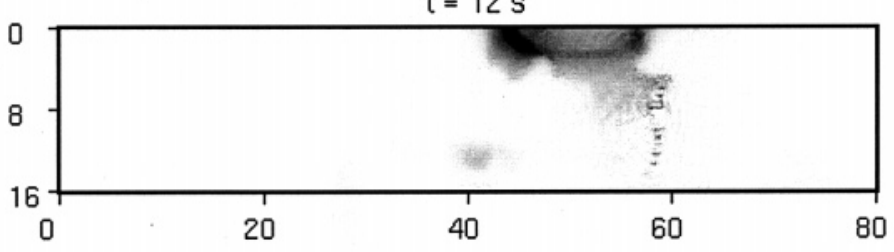

0

slip rate (m/s)

1.1 
geometries and more complex stress distributions. The fact that we have a bifurcation in the behavior of the system means that we must have a single nondimensional control parameter that determines whether a rupture will grow or stop. The determination of such a number can be very complex as shown by SCHMITTBUHL et al. (1996) who carried out an exhaustive study of the parameters' space of the BURRIDGE and KNOPOFF (1967) model. In the rectangular barrier and asperity models the stress field is uniform such that we expect no fractional powers; for this reason we successfully applied the simple dimensional analysis approach to find nondimensional numbers (see, e.g., LANDAU and LifSHITZ, 1959, page 63).

Following IDA (1972) and ANDREws (1976) we can easily show that the nondimensional number $\kappa$ derives from the competition between a measure of the strain energy released during rupture and the energy dissipated in fracture. Rupture resistance, or energy release rate for our slip-weakening friction model (1) is

$$
G=\frac{1}{2} T_{u} D_{c}
$$

Strain energy change $\Delta U$ per unit fault surface in a rupture zone of characteristic length $W$ is on the order of:

$$
\Delta U=\frac{1}{2}\langle D\rangle T_{e} \simeq A T_{e}^{2} / \mu R
$$

where $\langle D\rangle \simeq T_{e} / \mu W$ is the average slip on a fault of length $W, T_{e}$ is the effective stress drop and $A$ a numerical coefficient on the order of 1 . Thus, our definition (5) implies that

$$
\kappa \simeq \Delta U / G
$$

For simple uniformly-stressed models, ANDREws' (1976) critical rupture patch implies that $\kappa_{c} \simeq A$. The critical value is simply the numerical coefficient $A$ that relates these two measures of energy. As we mentioned earlier, it is more difficult to estimate the critical value for faults in $3-\mathrm{D}$, because the shape of the fault and the rupture mode will produce variations in $A$ that are not easy to calculate. For the long rectangular fault we prefer the determination of Figure 5 which yields $\kappa=0.8$. For the asperity we found $\kappa=0.7$.

In a more realistic situation, as for the Landers earthquake, stress is complex and we expect the numerical value of $\kappa_{c}$ to be different because somehow the measure of energy must integrate spatial variations of stress. We believe, however, that this number should be of order 1 as we obtained in our analysis. For more general models with fractal distributions of stress and fracture resistance $G$, we expect that the simple expression for $\kappa$ may include fractional powers.

\section{Figure 8}

Snapshots of the slip rate field on the fault of the Landers 1992 earthquake computed at regular intervals of $2 \mathrm{~s}$, starting with the initial stress field shown in the upper snapshot of Figure 7. 
We can now speculate on the behavior of active faults in the earth. We have seen from these simple examples that the essential requirement for rupture growth is that $\kappa>\kappa_{c}$ where the critical value of $\kappa$ depends on the geometry of the problem but is of order 1. Once $\kappa$ becomes substantially larger than critical, ruptures tend to become super-shear very rapidly although this is rarely observed in nature. Why is that? We believe that ruptures are in general sub-shear because faults always stay close to the critical condition. The reason may be very simple: as soon as the conditions for rupture in a fault area are fulfilled, a rupture will occur unloading the fault. When the system is reloaded to criticality another earthquake will occur and so on. The size of these earthquakes is not determined by the load but by the connectivity of the stress or rupture resistance field. In other words, the earthquake will propagate to the extent the stress field permits! Under special circumstances stress can be high and the fault remains in supercritical condition. Only in those exceptional circumstances will super-shear speeds be observed (e.g., ArCHUlETA, 1984). The normal behavior for earthquakes is to break under critical conditions at speeds below the shear wave speed. This eliminates the longstanding dilemma of why earthquakes stop, and why there are earthquakes of vastly different sizes on the same fault segment.

\section{Conclusions}

We have carefully studied rupture growth on a simple flat fault embedded in a homogeneous elastic medium of rigidity $\mu$. It emerges from our studies that rupture is controlled by a nondimensional number $\kappa=T_{\mathrm{e}}^{2} / \mu T_{u} \times L / D_{c}$ where $L$ is a characteristic size of the stress field, for instance the patch (asperity) radius or the width of the fault or asperity, $T_{e}$ is a characteristic stress load on this patch, and $T_{u} \times D_{c}$ is a measure of energy release rate on the fault.

We then estimated $\kappa$ for the dynamic simulation of the 1992 Landers earthquake by Olsen et al. (1997) that was based on the WALD and Heaton (1994) kinematic model. From a rough estimate we found that $\kappa$ was slightly less than 1 , implying that the Landers earthquake rupture occurred under conditions that were almost critical. Considerable additional evidence in favor of criticality was examined, leading to the hypothetical suggestion that earthquake ruptures occur as soon as the stress distribution on the fault becomes critical and that faults rarely stay in a meta-stable state that would allow large super-critical states on the fault.

In our analysis to date we have assumed uniform rupture resistance (constants $T_{u}$ and $D_{c}$ ). All the complexity in our models arises from the heterogeneity of initial stress. As important as stress heterogeneity is probably the small-scale geometry of faulting. Its integration in fault models is difficult because most of the observations of fault rupture are still limited to the range of frequencies less than $1 \mathrm{~Hz}$ or about $3 \mathrm{~km}$ wavelength. This is too coarse a resolution to observe effects of complex 
geometry other than major fault segmentation as in Landers or Kobe. High-frequency seismic radiation is probably the only source of information regarding small-scale geometry.

The implications of this simple number are wide ranging and require extensive tests and analysis of modeling. We are currently carrying out such tests for the case of the Landers earthquake.

\section{Acknowledgements}

We thank J. R. Rice for very useful discussions concerning the origin of the nondimensional number $\kappa$. The computations in this study were carried out in the parallel computer of Département de Modélisation Physique et Mathématique of Institut de Physique du Globe de Paris. R. Madariaga's work was supported by the Environment Program of the European Community under project SGME and by CNRS (Centre National de la Recherche Scientifique) under contract 99PNRN13AS of the PNRN program. K. Olsen's work was supported by NSF Grant EAR 9628682 and by the Southern California Earthquake Center (SCEC). SCEC is funded by NSF Cooperative Agreement EAR-8920136 and USGS Cooperative Agreements 14-08-0001-A0899 and 1434-HQ-97AG01718. This is ICS contribution 0345-93EQ and SCEC contribution number 529.

\section{REFERENCES}

AKI, K. (1979), Characterization of Barriers of an Earthquake Fault, J. Geophys. Res. 84, 6140-6148. Andrews, J. (1976), Rupture Velocity of Plane Strain Shear Cracks, J. Geophys. Res. 81, 5679-5687.

ArChuletA, R. (1984), A Faulting Model for the 1979 Imperial Valley Earthquake, J. Geophys. Res. 89, 4559-4585.

Beroza, G., and Mikumo, T. (1996), Short Slip Duration in Dynamic Rupture in the Presence of Heterogeneous Fault Properties, J. Geophys. Res. 101, 22,449-22,460.

Bouchon, M. (1997), The State of Stress on Some Faults of the San Andreas System as Inferred from Near-field Strong Motion Data, J. Geophys. Res. 102, 11,731-11,744.

Burridge, R., and Knopoff, L. (1967), Model and Theoretical Seismicity, Bull. Seismol. Soc. Am. 67, $341-371$.

Carlson, J., and Langer, J. (1989), Mechanical Model of an Earthquake Fault, Phys. Rev. A40, $6470-6484$.

Cochard, A., and Madariaga, R. (1994), Dynamic Faulting under Rate-dependent Friction, Pure appl. geophys. 142, 419-445.

Cochard, A., and Madariaga, R. (1996), Complexity of Seismicity due to Highly Rate-dependent Friction, J. Geophys. Res. 101, 17,581-17,596.

CoHeE, B., and BerozA, G. (1994), Slip Distribution of the 1992 Landers Earthquake and its Implications for Earthquake Source Mechanics, Bull. Seismol. Soc. Am. 84, 692-712.

Cotton, F., and Campillo, M. (1995), Frequency Domain Inversion of Strong Motions: Application to the 1992 Landers Earthquake, J. Geophys. Res. 100, 3961-3975.

DAs, S. (1981), Three-dimensional Spontaneous Rupture Propagation and Implications for the Earthquake Source Mechanism, Geophys. J. Roy. astr. Soc. 67, 375-393. 
DAS, S., and AKI, K. (1977a), A Numerical Study of Two-dimensional Spontaneous Rupture Propagation, Geophys. J. Roy. astr. Soc. 50, 643-668.

Das, S., and AKI, K. (1977b), Fault Plane with Barriers: A Versatile Earthquake Model, J. Geophys. Res. $82,5658-5670$.

DAs, S., and Kostrov, D. (1983), Breaking of a Single Asperity: Rupture Process and Seismic Radiation, J. Geophys. Res. 88, 4277-4288.

DAY, S. (1982), Three-dimensional Simulation of Spontaneous Rupture: The Effect of Non-uniform Prestress, Bull. Seismol. Soc. Am. 72, 1881-1902.

Dieterich, J. (1978), Time-dependent Friction and the Mechanics of Stick-slip, Pure appl. geophys. 116, $792-806$.

Funuyama, E., and Madariaga, R. (1998), Rupture Dynamics of a Planar Fault in a 3-D Elastic Medium: Rate- and Slip-weakening Friction, Bull. Seismol. Soc. Am. 88, 1-17.

Griffith, A. A. (1929), The Phenomenon of Rupture and Flow in Solids, Phil. Trans. Roy. Soc. London A221, 163-198.

Harris, R., and Day, S. (1993), Dynamics of Fault Interaction: Parallel Strike-slip Faults, J. Geophys. Res. 98, 4461-4472.

Heaton, T. (1990), Evidence for and Implications of Self-healing Pulses of Slip in Earthquake Rupture, Phys. Earth. Planet. Int. 64, 1-20.

Husseini, M. I., Jovanovich, D. B., Randall, M. J., and Freund, L. B. (1975), The Fracture Energy of Earthquakes, Geophys. J. Roy. astr. Soc. 43, 367-385.

IDA, Y. (1972), Cohesive Force across the Tip of a Longitudinal-shear Crack and Griffith's Specific Surface Energy, J. Geophys. Res. 77, 3796-3805.

Ide, S., and TAKeo, M. (1997), Determination of the Constitutive Relation of Fault Slip Based on Wave Analysis, J. Geophys. Res. 102, 27,379-27,391.

Kanamori, H., and Stewart, G. S (1976), Seismological Aspects of the Guatemala Earthquake of February 4, 1976, J. Geophys. Res. 83, 3427-3434.

Kostrov, B. (1964), Self-similar Problems of Propagation of Shear Cracks, J. Appl. Math. Mech. 28, $1077-1087$.

Kostrov, B., and Das, S., Principles of Earthquake Source Mechanics (Cambridge University Press, Cambridge, UK 1989).

Landau, L., and Lifshitz, E., Fluid Mechanics (Pergamon Press, London 1959).

Madariaga, R. (1976), Dynamics of an Expanding Circular Fault, Bull. Seismol. Soc. Am. 66, 639-667.

MadariagA, R. (1979), On the Relation between Seismic Moment and Stress Drop in the Presence of Stress and Strength Heterogeneity, J. Geophys. Res. 84, 2243-2250.

Madariaga, R., Olsen, K. B., and Archuleta, R. J. (1998), Modeling Dynamic Rupture in a 3-D Earthquake Fault Model, Bull. Seismol. Soc. Am. 88, 1182-1197.

Madariaga, R., Olsen, K. B., and Peyrat, S. (2000), Rupture Dynamics in 3-D: A Review, Annali di Geofisica, accepted for publication.

Mikumo, T., and Miyatake, T. (1995), Heterogeneous Distribution of Dynamic Stress Drop and Relative Fault Strength Recovered from the Results of Waveform Inversion, Bull. Seismol. Soc. Am. 85, $178-193$.

Okubo, P. (1989), Dynamic Rupture Modeling with Laboratory-derived Constitutive Relations, J. Geophys. Res. 94, 12,321-12,335.

Ohnaka, M., and Shen, L.-F. (1999), Scaling of Rupture Process from Nucleation to Dynamic Propagation: Implications of Geometric Irregularity of the Rupturing Surfaces, J. Geophys. Res. 104, $817-844$.

Olsen, K., Madariaga, R., and Archuleta, R. (1997), Three-dimensional Dynamic Simulation of the 1992 Landers Earthquake, Science 278, 834-838.

Rice, J. R. (1993), Spatio-temporal Complexity of Slip on a Fault, J. Geophys. Res. 88, 9885-9907.

Rice, J., and Ben-Zion, Y. (1996), Slip Complexity in Earthquake Fault Models, Proc. Natl. Acad. Sci. USA 93, 3811-3818.

SHAw, B., and RicE, J. R. (2000), Existence of Continuum Complexity in the Elastodynamics of Repeated Fault Ruptures, J. Geophys. Res., in press. 
Schmittbuhl, J., Vilotte, J. P., and Roux, S. (1996), A Dissipation-based Analysis of an Earthquake Fault Model, J. Geophys. Res. 101, 27,741-27,764.

Wald, D., and Heaton, T. (1994), Spatial and Temporal Distribution of Slip for the 1992 Landers, California Earthquake, Bull. Seismol. Soc. Am. 84, 668-691.

(Received August 4, 1999, revised April 4, 2000, accepted April 5, 2000) 